\title{
Avaliação Institucional de Planejamentos de IMRT nos Termos Recomendados pelo ICRU 83
} Institutional Evaluation of IMRT Planning in Terms Recommended by ICRU 83

\author{
Marmitt. Camila ${ }^{1}$, Mantovani. C. M. Camila ${ }^{2}$, Fernandes. C. O. Tatiane ${ }^{3}$ \\ ${ }^{1}$ Liga Paranaense de Combate ao Câncer, Hospital Erasto Gaertner, Curitiba, Brasil \\ ${ }^{2}$ Departamento de Radioterapia, Liga Paranaense de Combate ao Câncer, Hospital Erasto Gaertner, \\ Curitiba, Brasil \\ ${ }^{3}$ Departamento de Radioterapia, Liga Paranaense de Combate ao Câncer, Hospital Erasto Gaertner,
} Curitiba, Brasil

\begin{abstract}
Resumo
Uma das técnicas empregadas atualmente para o tratamento radioterápico e a que oferece uma dose maior na área desejada juntamente com uma maior conformação de dose somente no alvo, é a radioterapia de intensidade modulada (Intensity Modulated Radiation Therapy - IMRT). A publicação "The International Commission on Radiation Units and Measurements (ICRU)" através do Report 83 descreve recomendações de procedimentos, prescrição, registro e notificações de planejamentos IMRT. O documento recomenda a avaliação e registro do plano através de parâmetros como: dose quase mínima (D98\%, D95\%), dose média (D50\%), dose quase máxima (D2\%), do índice de homogeneidade (IH) e índice de conformidade (IC), entre outros, dados estes avaliados em detrimento da porcentagem de volume do alvo com a dose recebida. O presente estudo busca analisar retrospectivamente planejamentos realizados na instituição a fim de analisar a necessidade de implementação de novos parâmetros de avaliação de planejamentos e criação de uma ficha de análise de DVH (Dose Volume Histogram) para registro dos mesmos.
\end{abstract}

Palavras-Chave: Física Médica; Radioterapia.

\begin{abstract}
One of the techniques currently employed for radiotherapy treatment, which offers a higher dose intensity in the desired area along with a higher target-only dose conformation, is Intensity Modulated Radiation Therapy (IMRT). The publication "The International Commission on Radiation Units and Measurements (ICRU)" through Report 83 describes recommendations for procedures, prescription, registration and notifications of IMRT planning. The document recommends the evaluation and registration of the plan through parameters such as: almost minimum dose (D98\%, D95\%), average dose (D50\%), almost maximum dose (D2\%), homogeneity index and compliance, among others, data evaluated at the expense of the target volume percentage with the dose received. The present study seeks to analyze retrospectively the plans made at the institution in order to analyze the need to implement new parameters for the evaluation of plans and the creation of a DVH (Dose Volume Histogram) analysis form to record them.
\end{abstract}

Key-words: Medical Physics; Radiation Therapy.

\section{Introdução}

A utilização de novas técnicas de tratamentos em radioterapia está crescente, novas descobertas de algoritmos de otimização dos planos de tratamento, técnicas de modulação do feixe de radiação e conformação da dose fazem com que se alcance com mais precisão a destruição das células tumorais e preservação dos tecidos sadios.

A partir do desenvolvimento da tomografia computadorizada e da utilização das imagens em planejamentos radioterápicos - radioterapia tridimensional conformacional, os radio-oncologistas puderam ter uma melhor precisão da localização do tumor e dos órgãos adjacentes quando comparada à técnica bidimensional (2D) que utiliza apenas imagens radiográficas e referências ósseas para planejamento radioterápico. Porém a entrega da dose no volume alvo sem comprometimento dos órgãos adjacentes ainda é um desafio, devido às limitações de colimação de volumes irregulares. Após o surgimento das imagens tridimensionais (3D) e com o avanço da tecnologia computacional começou-se a desenvolver novas técnicas de conformação da dose, em que sua maior concentração chega no volume alvo passando pelos tecidos sadios e provocando o menor dano possível.

A Radioterapia Tridimensional Conformacional (Conformal Radiation Therapy - 3D-CRT) é a técnica de tratamento baseada em informações anatômicas tridimensionais e com distribuições de dose que se conformam o mais estreitamente possível ao volume alvo em termos de dose 
adequada ao tumor com o mínimo possível de dose em tecidos normais. O conceito de distribuição de dose conformacional também inclui objetivos clínicos tais como maximizar a probabilidade de controle tumoral (Tumoral Control Probability - TCP) e minimizar a probabilidade de complicação de tecido normal (Normal Tissue Control Probability - NTCP). Assim, a técnica 3D-CRT engloba tanto raciocínio clínico quanto biológico para alcançar os objetivos desejados.

Uma das técnicas empregada atualmente e a que oferece uma maior intensidade de dose na área desejada juntamente com uma maior conformação de dose somente no alvo, é a radioterapia de intensidade modulada (Intensity Modulated Radiation Therapy - IMRT). Esta técnica, diferentemente da 3D - CRT tradicional libera múltiplos feixes com intensidades não uniformes, permitindo uma maior conformação da radiação em torno da geometria do volume alvo, diminuindo assim, a dose nos tecidos e órgãos adjacentes.

A forma de planejamento da técnica de IMRT consiste na predeterminação dos parâmetros dosimétricos, tanto do volume alvo como dos órgãos que se deseja proteger, o que chamamos de planejamento inverso (inverse planning). Dessa forma um software de planejamento através da otimização da dose e de tentativa e erro, cria um conjunto de fluências que julga ser a seleção mais favorável para o planejamento, porém muitas vezes é necessária a interferência manual para se alcançar o objetivo final.

Durante o planejamento do tratamento são gerados histogramas de dose-volume (Dose Volume Histogram - DVH) que demonstram a distribuição da dose por um determinado volume. A avaliação da dose no volume alvo, juntamente com outros parâmetros de observação, é que vai garantir se o planejamento está dentro do proposto pelo radiooncologista.

Considerando que o planejamento da técnica de radioterapia de IMRT é diferente da técnica 3D CRT tradicional, sua forma de avaliação do plano também necessita de outros critérios, dessa forma The International Commission on Radiation Units and Measurements (ICRU) através do Report 83 descreve recomendações de procedimentos, prescrição, registro e notificações de planejamentos IMRT.

O documento recomenda a avaliação e registro do plano através de parâmetros como: dose quase mínima (D98\%, D95\%), dose média (D50\%), dose quase máxima (D2\%), índice de homogeneidade (IH) e de conformidade (IC), entre outros. Estes dados avaliados em detrimento da porcentagem de volume do alvo com a dose recebida.

A homogeneidade e a conformidade da dose são especificações independentes da qualidade da distribuição da dose absorvida. A homogeneidade caracteriza a uniformidade da distribuição da dose absorvida dentro do volume alvo. A conformidade caracteriza o grau em que a região de alta dose está em conformidade com o volume alvo, geralmente o PTV (Planning Target Volume).

$\mathrm{O}$ índice de homogeneidade é definido pelo ICRU 83 de acordo com a seguinte equação (1):

$$
I H=\frac{D 2 \%-D 98 \%}{D 50 \%}
$$

Sendo que IH representa o índice de homogeneidade, D2\% a dose absoluta em $2 \%$ do volume alvo, D98\% a dose absoluta em $98 \%$ do volume alvo e D50\% a dose absoluta em $50 \%$ do volume alvo.

$O$ índice do conformidade (IC) é definido pelo ICRU 83 sendo a razão entre o volume da dose prescrita de tratamento $\left(V_{v t}\right)$ e o volume do PTV $\left(V_{P T V}\right)$ de acordo com a equação (2), este valor é fornecido pela grande maioria dos sistemas de planejamento.

$$
I C=\frac{V_{v t}}{V_{P T V}}
$$

De acordo com o ICRU 83 um índice de homogeneidade igual a 0 (zero) indica que a distribuição da dose absorvida está homogênea e um índice de conformidade igual a 1 (um) indica que a distribuição da dose absorvida está conformada.

Estes são alguns dos parâmetros recomendados pelo ICRU 83 e selecionados para verificação no presente trabalho, lembrando que o ICRU 83 é uma forma de induzir a uma padronização internacional de termos, registro e prescrições, ou seja, é um documento de recomendação e não uma normativa, não se tornando obrigatório seguir em todas as instituições.

\section{Materiais e Métodos}

Para análise dos dados, foram avaliados 78 planos de IMRT de duas regiões anatômicas específicas, próstata e cabeça e pescoço, realizados durante o período de janeiro de 2015 até agosto de 2016 no Hospital Erasto Gaertner em Curitiba, Brasil. Os planejamentos foram realizados utilizando a técnica IMRT com feixes de fótons com energia de $6 \mathrm{MV}$, em aceleradores lineares do tipo AL $600 \mathrm{CD}$ e Clinac 2100, ambos fabricados pela empresa Varian Medical Systems, Palo Alto CA.

Foram analisados seus respectivos DVHs através do sistema de planejamento Eclipse (Versão 13.5) do fabricante Varian. Através dos DVHs foi possível coletar as informações de dose e volume para posterior análise.

Sendo assim, para cada um dos planejamentos, foram avaliados as porcentagens da dose absoluta para $98 \%, 95 \%, 50 \%$ e $2 \%$ do volume do PTV e listadas na planilha. Assim foi possível determinar também o quanto a dose nos volumes alvos (PTVs) está conformada e homogênea, avaliando os índices de conformidade e homogeneidade recomendados pelo ICRU 83. Para determinação do $\mathrm{IH}$ foi utilizado a equação 1 e para o IC foi utilizado o valor determinado pelo sistema de planejamento. 
$\mathrm{Na}$ rotina atual da Instituição avaliam-se os planejamentos IMRT de acordo com a prescrição mínima de $100 \%$ da dose em $95 \%$ do volume alvo, as doses limites em órgãos de risco circunvizinhos, a conformidade das curvas de isodose em todos os cortes da tomografia, os valores de dose máxima e mínima e o DVH do plano, entre outros parâmetros de avaliação. Todos esses dados são repassados ao radio-oncologista que então libera ou não o início do tratamento. No entanto, o registro desses dados fica restrito ao sistema de planejamento e à impressão do DVH do plano.

A partir do estudo retrospectivo destes planejamentos está proposta a criação de uma Ficha de Análise de DVH para IMRT, onde estes dados analisados poderão ser registrados e posteriormente arquivados ao prontuário de cada paciente.

O presente estudo foi submetido ao Comitê de Ética e Pesquisa do Hospital Erasto Gaertner - Liga Paranaense de Combate ao Câncer com número de Certificado de Apresentação para Apreciação Ética (CAAE): 66517917.5.0000.0098.

\section{Resultados}

Foram avaliados 35 planejamentos de próstata e 43 planejamentos de cabeça e pescoço, totalizando 78 planos utilizando a técnica IMRT.

Para 0 índice de homogeneidade de planejamentos de próstata $54,3 \%$ dos resultados estão na faixa de 0,11 e 0,2 conforme Gráfico 1 abaixo. Para cabeça e pescoço $51,2 \%$ dos planejamentos obtiveram índice de homogeneidade na faixa de 0 a 0,1 e $48,8 \%$ entre 0,11 a 0,2 conforme Gráfico 2.

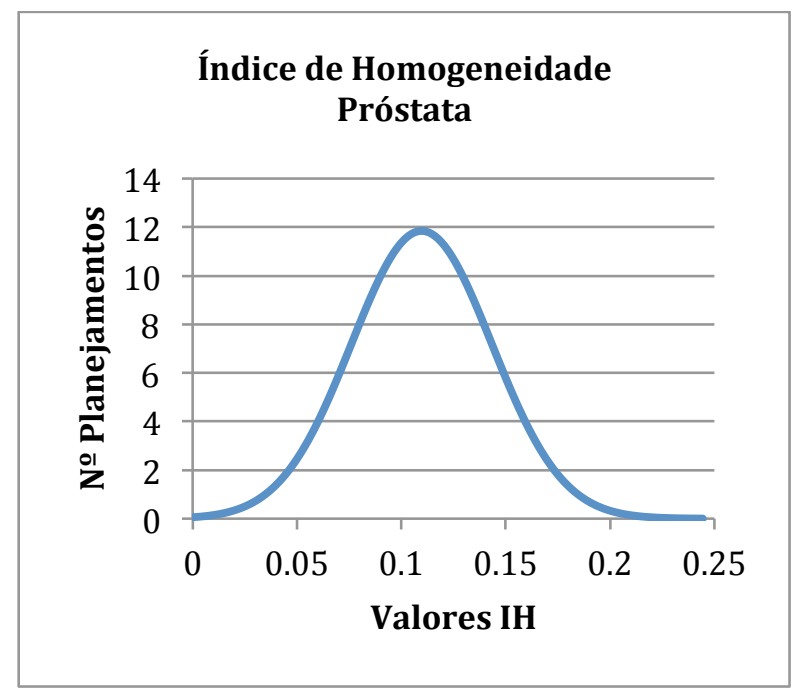

Gráfico 1: Índice de Homogeneidade de Planejamentos de Próstata

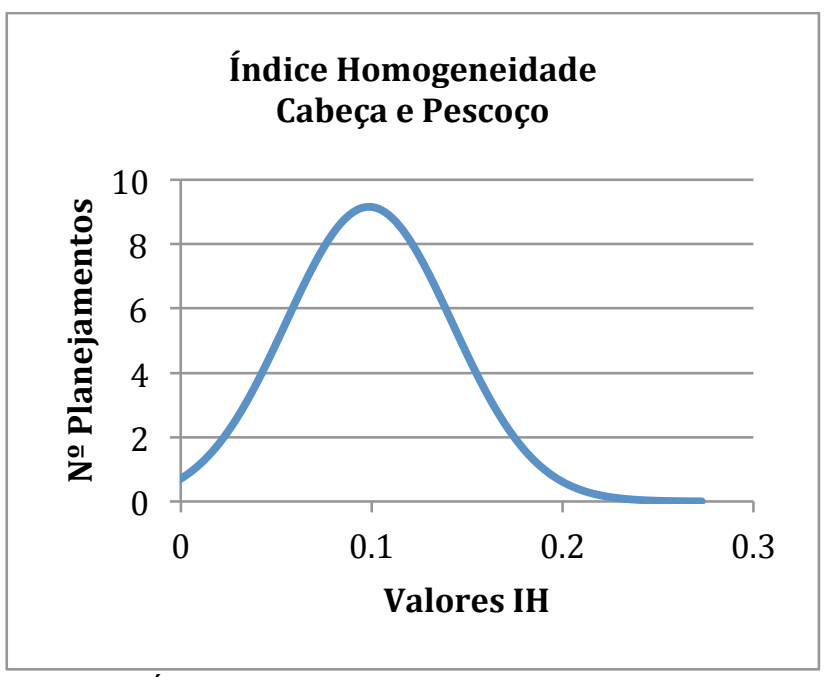

Gráfico 2: Índice de Homogeneidade de Planejamentos de Cabeça e Pescoço

Para o índice de conformidade, $37,1 \%$ dos planejamentos de próstata estavam na faixa de 1,01 e 1,1, e 34,28\% dos planejamentos entre 1,11 e 1,5 conforme Gráfico 3. Para cabeça e pescoço $55,8 \%$ dos planejamentos estavam na faixa entre os valores de 1,11 a 1,5 conforme Gráfico 4 .

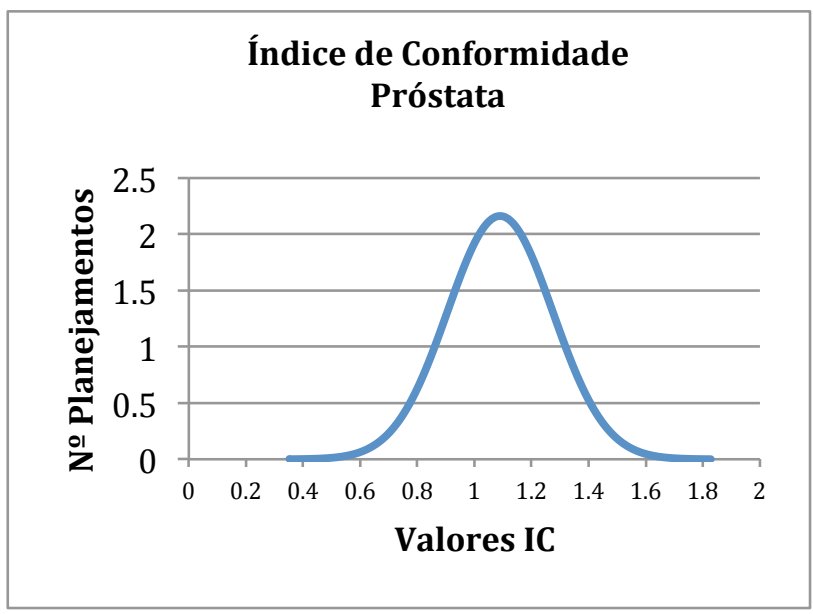

Gráfico 3: Índice de Conformidade de Planejamentos de Próstata

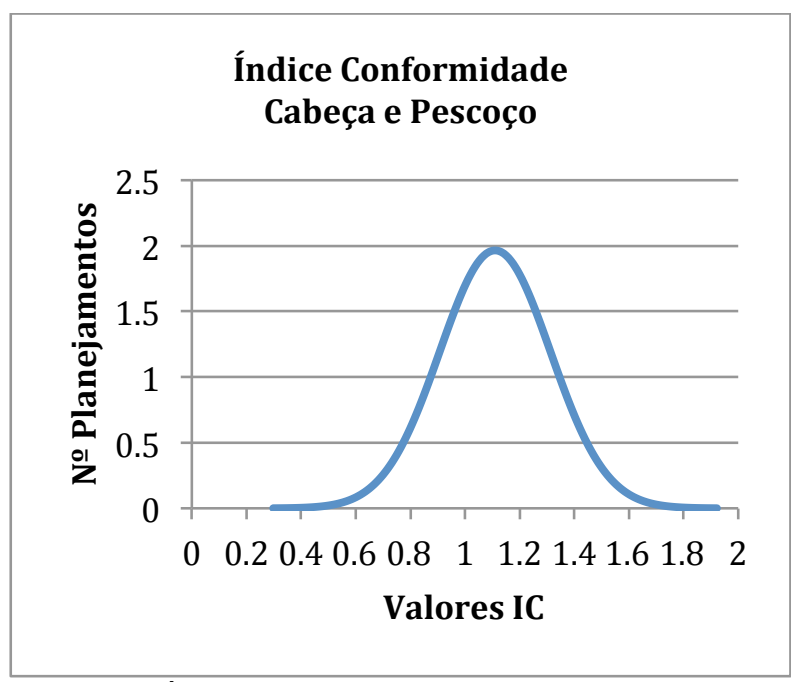

Gráfico 4: Índice de Conformidade de Planejamentos de Cabeça e Pescoço 
A partir desses resultados, podemos então utilizá-los para nos ajudar a ter um melhor julgamento dos planos posteriores, ou seja, sabendo os valores de maior incidência contidos em nossa biblioteca, podemos verificar se é possível estar melhorando ainda mais o plano ou se o mesmo encontra-se dentro de um valor satisfatório para a instituição, tendo em vista, que o ICRU não define os valores limites para índice de conformidade e homogeneidade, porém, podemos nos basear em dados já existentes para definir um valor satisfatório ou não para planejamentos realizados na instituição.

Sendo assim, como sugestão, extraímos os valores a partir do ideal para mais ou para menos, até um limite onde a maioria dos planejamentos alcançou. Para índice de homogeneidade de próstata podemos dizer que o aceitável seria a partir de 0 o qual seria o ideal, até 0,15 , o qual é um valor dentro do intervalo onde a maioria dos planejamentos estavam enquadrados, para índice de homogeneidade de cabeça e pescoço o valor seria na faixa de 0 a 0,1 . Para o índice de conformidade de planejamentos de próstata, extraímos um valor favorável de 0,8 a 1,4 e para planejamentos de cabeça e pescoço na faixa de 0,8 a 1,5. Tendo em vista o objetivo principal é claro, de conseguir a melhor homogeneidade e conformidade possível dentro dos padrões do ICRU 83.

\section{Conclusão}

$O$ resultado foi satisfatório, tendo em vista que o estudo foi retrospectivo e que os planejamentos realizados alcançaram valores muito próximos do ideal, sendo que um índice de homogeneidade igual a 0 (zero) indica que a distribuição da dose absorvida está homogênea e um índice de conformidade igual a 1 (um) indica que a distribuição da dose absorvida está conformada.

Sendo assim, como recomenda o ICRU 83 criou-se a Ficha de Análise de DVH para IMRT conforme Figuras 1 e 2 para próstata e cabeça e pescoço respectivamente, como sugestão de registro dessas informações para futuros planejamentos realizados na Instituição, criando assim uma biblioteca de valores para posteriores consultas e também auxiliar na revisão de planos futuros.

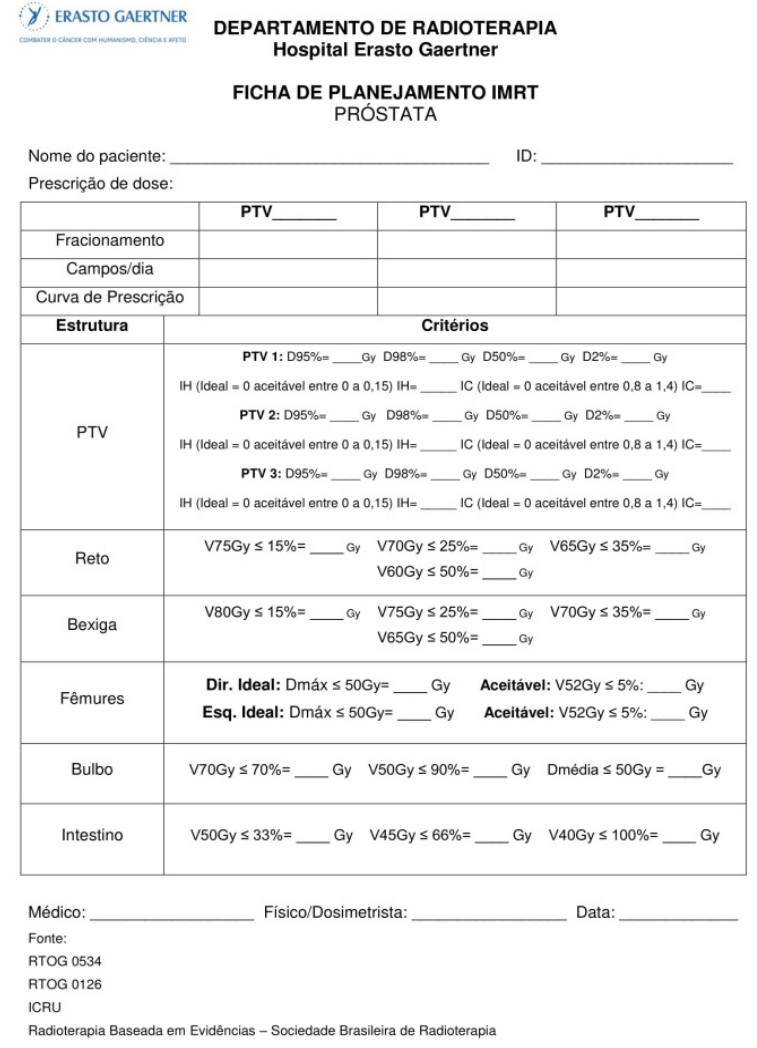

Figura 1: Modelo de ficha de análise de DVH para IMRT de planejamento de próstata proposta para implementação no departamento de radioterapia da instituição.

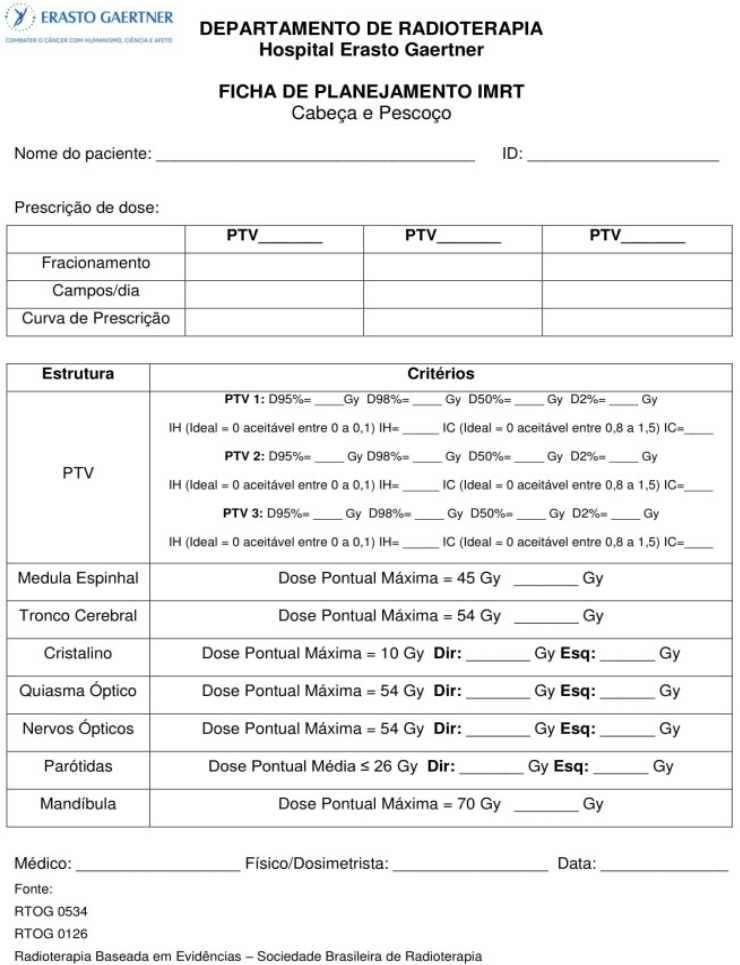

Figura 2: Modelo de ficha de análise de DVH para IMRT de planejamento de cabeça e pescoço proposta para implementação no departamento de radioterapia da instituição. 


\section{Referências}

1. Mayles, Philip; Nahum, Alan; Rosenwald, Jean-Claude (Ed.) Handbook of radiotherapy physics: theory and practice. CRC Press, 2007

2. Khan, Faiz M.; Gerbi, Bruce John. Treatment planning in radiation oncology. WoltersKluwer Health/Lippincott Williams \&Wilkins, 2012.

3. Brady, Luther W.; Heilmann, H. P.; Molls, M. New technologies in radiation oncology. Springer, 2006

4. Mundt, Arno J.; Roeske, John C. Intensity modulated radiation therapy: a clinical perspective. PMPH-USA, 2005.

5. Meyer, John L. et al. IMRT· IGRT. SBRT. 2006.

6.ICRU Report 83. Prescribing, Recording, and Reporting Photon-Beam Intensity-Modulated Radiation Therapy (IMRT). Journal of the International Commission on Radiation Units and Measurements, Volume 10, Issue 1, 1 April 2010, Pages NP, https://doi.org/10.1093/jicru/10.1.Report83.

\section{Contato:}

Camila Marmitt

Hospital Erasto Gaertner - R. Dr. Ovande do Amaral, 201 - Jardim das Americas, Curitiba - PR, 81520060

camilamarmitt@yahoo.com.br 